\title{
Stereo vision-based variational optical flow estimation
}

\author{
P. V. Belyakov ${ }^{1}, M$. B. Nikiforov ${ }^{1,}{ }^{*}, E . R$. Muratov $^{1}$, and $O . V$. Melnik $^{1}$ \\ ${ }^{1}$ Ryazan state radio engineering university named after V.F. Utkin, 59/1, Gagarina str, Ryazan, \\ 390005, Russia
}

\begin{abstract}
Optical flow computation is one of the most important tasks in computer vision. The article deals with a modification of the variational method of the optical flow computation, according to its application in stereo vision. Such approaches are traditionally based on a brightness constancy assumption and a gradient constancy assumption during pixels motion. Smoothness assumption also restricts motion discontinuities, i.e. the smoothness of the vector field of pixel velocity is assumed. It is proposed to extend the functional of the optical flow computation in a similar way by adding a priori known stereo cameras extrinsic parameters and minimize such jointed model of optical flow computation. The article presents a partial differential equations framework in image processing and numerical scheme for its implementation. Performed experimental evaluation demonstrates that the proposed method gives smaller errors than traditional methods of optical flow computation.
\end{abstract}

\section{Introduction}

Variational methods of the optical flow computation are the most effective and accurate methods of the image pixels displacement estimating. The optical flow is essentially a movement velocity vector field of one image relative to another. Variational methods make it possible to integrate a different mathematical concepts into one computational framework. This property makes them especially attractive for use in various key areas of computer and technical vision [1].

Recently though network methods show best results in optical flow estimation they still need large and specific ground truth data sets and time-costly training cycles. So the variational methods of energy functional minimisation are still suitable for further improvement of optical flow estimation.

Similar to [2] our model relies on intrinsic and extrinsic stereo cameras parameters, but instead of optical flow estimation based on TV-L1 minimization proposed approach is based on the classical variational method of optical flow functional energy minimization.

\footnotetext{
* Corresponding author: nikiforov.m.b@evm.rsreu.ru
} 


\section{Optical flow constraint}

Recall variational model of optical flow computation is based on energy functional minimisation that consists of three basic assumptions. Brightness constancy is assumed that the pixel value is constant during its displacement. In the same way, assumed image gradient value does not vary due to the pixel displacement. And a third assumption is spatial-temporal smoothness of the optical flow field. So the variational optical flow constraint can be written as

$$
E_{O F}(\boldsymbol{w})=E_{D}(\boldsymbol{w})+\beta E_{S}(\boldsymbol{w}) .
$$

The brightness constancy assumption and the gradient constancy assumption are measured by the energy

$$
E_{D}(\boldsymbol{w})=\int_{\Omega}\left(\psi_{D}\left(|I(\boldsymbol{x}+\boldsymbol{w})-I(\boldsymbol{x})|^{2}+\alpha\left(|\nabla I(\boldsymbol{x}+\boldsymbol{w})-\nabla I(\boldsymbol{x})|^{2}\right)\right) d x\right.
$$

and the smoothness term reads

$$
E_{S}(\boldsymbol{w})=\int_{\Omega} \psi_{S}\left(|\nabla u|^{2},+|\nabla v|^{2}\right) d x
$$

where $I(x, y, t): \Omega \times[0, \infty) \rightarrow \boldsymbol{R}$ is the image sequence, $x=(x, y, 1)^{T}$ - pixel coordinates inside a rectangular area of the image $\Omega \subset \boldsymbol{R}^{2}$ and $\nabla I(\boldsymbol{x})$ is the image brightness gradient. The regularization parameter $\alpha>0$ is a coefficient that allows to control the effect of changes in brightness and brightness gradient on the functional (2) and accordingly (1). The regularization parameter $\beta>0$ describes how important is the smoothness requirement of the obtained displacement vector (3). Optical flow $\boldsymbol{w}=(u, v, 1)^{T}$ is the desired displacement vector between the pixels of two images. Penalty function $\psi\left(s^{2}\right)=\sqrt{s^{2}+\varepsilon^{2}}$ is same for both $\psi_{D}$ and $\psi_{S}$ and determines robustness of the functional (1) minimization to image brightness and flow field smoothness changes. For its differentiability, $\varepsilon$ is chosen sufficiently small [1].

\section{Camera pose constraint}

So it is proposed to modify one of the most accurate but at the same time most computationally complex variational method of optical flow computation (1), taking into account the known extrinsic and intrinsic parameters of the stereo cameras pair. The optical flow error correspondence between each pixel of two images can be corrected by using of reprojection error [3]

$$
S(\boldsymbol{X})=\left\|d\left(\boldsymbol{x}, P_{0} \boldsymbol{X}\right)\right\|^{2}+\|d(\boldsymbol{x}+\boldsymbol{w}, P \boldsymbol{X})\|^{2},
$$

where $d(\cdot)$ denotes the Euclidean distance between 3D points $\boldsymbol{X}$ projection to the image plane and its most likely true coordinate $\boldsymbol{x}$ on the image. Corresponding points in the image are connected with a point in $3 \mathrm{D}$ space through the $3 \times 4$ projection matrix $P_{0}$ and $P$ of the stereo pair cameras. Camera projection matrix $P_{0}=K[\boldsymbol{I} \mid \mathbf{0}]$ for image $I_{1}$, defined through the zero position vector $\mathbf{0}$ with unit rotation matrix $\boldsymbol{I}$. Rotation matrix $R$ and position vector $\boldsymbol{t}$ are the extrinsic stereo cameras parameters for the image $I_{2}$ defined as $R=\left[\begin{array}{lll}r_{11} & r_{12} & r_{13} \\ r_{21} & r_{22} & r_{23} \\ r_{31} & r_{32} & r_{33}\end{array}\right]$ and 
$\boldsymbol{t}=\left[\begin{array}{l}t_{x} \\ t_{y} \\ t_{z}\end{array}\right]$ respectively. Camera projection matrix $P=K[R \mid \boldsymbol{t}]$, accordingly $P=$ $\left[\begin{array}{llll}r_{11} & r_{12} & r_{13} & t_{x} \\ r_{21} & r_{22} & r_{23} & t_{y} \\ r_{31} & r_{32} & r_{33} & t_{z}\end{array}\right]$ and matrix $K$ is intrinsic camera parameters.

\section{A joint variational model}

Functional (1) is proposed to be supplemented by the term $E_{X}(\mathbf{w}, \mathbf{X})$, which takes into account the external parameters of the stereo pair cameras $[2,4]$. We define now optical flow functional optimization as

$$
\underset{\mathbf{w}, \mathbf{X}}{\arg \min } E(\mathbf{w}, \mathbf{X})=\underset{\mathbf{w}, \mathbf{X}}{\arg \min } E_{O F}(\mathbf{w})+\gamma E_{X}(\mathbf{w}, \mathbf{X}) .
$$

The reprojection error is included in the variational model for the optical flow computation (4) as

$$
\begin{gathered}
E_{X}(\boldsymbol{w}, \boldsymbol{X})=\int_{\Omega}\left(\psi_{X}\left(\left\|\left[x \boldsymbol{p}_{0}^{3}-\boldsymbol{p}_{0}^{1}\right]^{T}\left[\begin{array}{c}
\boldsymbol{X} \\
1
\end{array}\right]\right\|^{2}\right)+\psi_{X}\left(\left\|\left[y \boldsymbol{p}_{0}^{3}-\boldsymbol{p}_{0}^{2}\right]^{T}\left[\begin{array}{c}
\boldsymbol{X} \\
1
\end{array}\right]\right\|^{2}\right)\right. \\
\left.+\psi_{X}\left(\left\|\left[(x+u) \boldsymbol{p}^{3}-\boldsymbol{p}^{1}\right]^{T}\left[\begin{array}{c}
\boldsymbol{X} \\
1
\end{array}\right]\right\|^{2}\right)+\psi_{X}\left(\left\|\left[(y+v) \boldsymbol{p}^{3}-\boldsymbol{p}^{2}\right]^{T}\left[\begin{array}{c}
\boldsymbol{X} \\
1
\end{array}\right]\right\|^{2}\right)\right) d x
\end{gathered}
$$

where $P_{0}=\left[\mathbf{p}_{0}^{1} \mathbf{p}_{0}^{2} \mathbf{p}_{0}^{3}\right]^{T}, P=\left[\mathbf{p}^{1} \mathbf{p}^{2} \mathbf{p}^{3}\right]^{T}$. Regularization parameter $\gamma>0$ determines the influence of reprojection error on the general functional (4) and $\psi_{X}$ has the same meaning of penalty function $\psi$.

If $(\mathbf{w}, \mathbf{X})$ is a solution to the minimization problem (4), then it satisfies the Euler Lagrange equations with reflecting boundary conditions.

\subsection{Optical flow}

To minimize (5) with respect to the optical flow vector $\mathbf{w}=(u, v)$, the Euler - Lagrange equations takes the form

$$
\begin{gathered}
\left.\frac{\partial}{\partial u} \psi_{X}\left|\left(\left.\left[(x+u) \mathbf{p}^{3}-\mathbf{p}^{1}\right]^{T}\left[\begin{array}{c}
\mathbf{X} \\
1
\end{array}\right]\right|^{2}\right)=2 \psi_{X}^{\prime}\right|\left[(x+u) \mathbf{p}^{3}-\mathbf{p}^{1}\right]^{T}\left[\begin{array}{c}
\mathbf{X} \\
1
\end{array}\right] \mid\right) \cdot\left|\left[(x+u) \mathbf{p}^{3}-\mathbf{p}^{1}\right]^{T} \cdot\left[\begin{array}{c}
\mathbf{X} \\
1
\end{array}\right] \cdot\right|\left[\mathbf{p}^{3}\right]^{T}\left[\begin{array}{c}
\mathbf{X} \\
1
\end{array}\right] \mid, \\
\frac{\partial}{\partial v} \psi_{X}\left(\left.\left[(y+v) \mathbf{p}^{3}-\mathbf{p}^{2}\right]^{T}\left[\begin{array}{c}
\mathbf{X} \\
1
\end{array}\right]\right|^{2}\right)=2 \psi_{X}^{\prime}\left(\left.\left[(y+v) \mathbf{p}^{3}-\mathbf{p}^{2}\right]^{T}\left[\begin{array}{c}
\mathbf{X} \\
1
\end{array}\right]\right|^{2}\right) \cdot\left|\left[(y+v) \mathbf{p}^{3}-\mathbf{p}^{2}\right]^{T} \cdot\left[\begin{array}{c}
\mathbf{X} \\
1
\end{array}\right] \cdot\right|\left[\mathbf{p}^{3}\right]^{T}\left[\begin{array}{c}
\mathbf{X} \\
1
\end{array}\right] \mid \cdot \quad(6)
\end{gathered}
$$

And the final equations of the optical flow functional $(4)$ of $\mathbf{w}=(u, v)$ minimization obtained by adding right-hand side of (6) to the Euler - Lagrange equations given by [1]: 


$$
\begin{gathered}
0=\psi_{D^{\prime}}\left(I_{z}^{2}+\alpha\left(I_{x z}^{2}+I_{y z}^{2}\right)\right) \cdot\left(I_{x} I_{z}+\alpha\left(I_{x x} I_{x z}+I_{x y} I_{y z}\right)\right) \\
-\beta \operatorname{div}\left(\psi_{S^{\prime}}\left(|\nabla u|^{2}+|\nabla v|^{2}\right) \nabla u\right) \\
+\gamma \psi_{X^{\prime}}\left(\left|\left[(x+u) \boldsymbol{p}^{3}-\boldsymbol{p}^{1}\right]^{T}\left[\begin{array}{c}
\boldsymbol{X} \\
1
\end{array}\right]\right|^{2}\right) \cdot\left|\left[(x+u) \boldsymbol{p}^{3}-\boldsymbol{p}^{1}\right]^{T} \cdot\left[\begin{array}{c}
\boldsymbol{X} \\
1
\end{array}\right]\right| \cdot\left|\left[\boldsymbol{p}^{3}\right]^{T}\left[\begin{array}{c}
\boldsymbol{X} \\
1
\end{array}\right]\right|, \\
0=\psi_{D^{\prime}}\left(I_{z}^{2}+\alpha\left(I_{x z}^{2}+I_{y z}^{2}\right)\right) \cdot\left(I_{y} I_{z}+\alpha\left(I_{y y} I_{y z}+I_{x y} I_{x z}\right)\right) \\
-\beta \operatorname{div}\left(\psi_{S^{\prime}}\left(|\nabla u|^{2}+|\nabla v|^{2}\right) \nabla v\right) \\
+\gamma \psi_{X^{\prime}}\left(\left|\left[(y+v) \boldsymbol{p}^{3}-\boldsymbol{p}^{1}\right]^{T}\left[\begin{array}{c}
\boldsymbol{X} \\
1
\end{array}\right]\right|^{2}\right) \cdot\left|\left[(y+v) \boldsymbol{p}^{3}-\boldsymbol{p}^{2}\right]^{T} \cdot\left[\begin{array}{c}
X \\
1
\end{array}\right]\right| \cdot\left|\left[\boldsymbol{p}^{3}\right]^{T}\left[\begin{array}{c}
X \\
1
\end{array}\right]\right|
\end{gathered}
$$

Here the same abbreviations for the partial derivatives and the temporal differences are used as in [1]:

$$
\begin{gathered}
I_{x}=\partial_{x} I(\boldsymbol{x}+\boldsymbol{w}), \\
I_{y}=\partial_{y} I(\boldsymbol{x}+\boldsymbol{w}), \\
I_{z}=I(\boldsymbol{x}+\boldsymbol{w})-I(\boldsymbol{x}), \\
I_{x x}=\partial_{x x} I(\boldsymbol{x}+\boldsymbol{w}), \\
I_{x y}=\partial_{x y} I(\boldsymbol{x}+\boldsymbol{w}), \\
I_{y y}=\partial_{y y} I(\boldsymbol{x}+\boldsymbol{w}), \\
I_{x z}=\partial_{x} I(\boldsymbol{x}+\boldsymbol{w})-\partial_{x} I(\boldsymbol{x}), \\
I_{y z}=\partial_{y} I(\boldsymbol{x}+\boldsymbol{w})-\partial_{y} I(\boldsymbol{x}) .
\end{gathered}
$$

Using standard discretization for the derivatives, the resulting sparse linear systems of equations (7) can be solved with common numerical methods, such as Gauss-Seidel or SOR iterations:

$$
A \boldsymbol{x}=\boldsymbol{b}, A=V+D+U, D x^{(m+1)}=(1-\omega) D x^{m}-\omega\left(V x^{(m+1)}+U x^{m}\right)+\omega b,
$$

where $V$ - a lower triangular matrix, $U-$ an upper triangular matrix, $D-$ a diagonal matrix и $\omega-$ a relaxation factor [5].

Denote

$$
\begin{gathered}
I_{\nabla}=\left(I_{x}, I_{y}, I_{z}\right)^{T}, S=I_{\nabla} I_{\nabla}^{T}, T=I_{\nabla x} I_{\nabla x}^{T}+I_{\nabla y} I_{\nabla y}^{T}, \\
a=\left|\left[\boldsymbol{p}^{3}\right]^{T}\left[\begin{array}{c}
\boldsymbol{X} \\
1
\end{array}\right]\right|, \\
q_{1}=\left|\left[x \boldsymbol{p}^{3}-\boldsymbol{p}^{1}\right]^{T}\left[\begin{array}{c}
\boldsymbol{X} \\
1
\end{array}\right]\right| a=\left|\left[x \boldsymbol{p}^{3}-\boldsymbol{p}^{1}\right]^{T} \cdot\left[\begin{array}{c}
\boldsymbol{X} \\
1
\end{array}\right]\right|\left|\left[\boldsymbol{p}^{3}\right]^{T}\left[\begin{array}{c}
\boldsymbol{X} \\
1
\end{array}\right]\right|, q_{2}=\left|\left[y \boldsymbol{p}^{3}-\boldsymbol{p}^{2}\right]^{T}\left[\begin{array}{c}
\boldsymbol{X} \\
1
\end{array}\right]\right| a= \\
\left|\left[y \boldsymbol{p}^{3}-\boldsymbol{p}^{2}\right]^{T}\left[\begin{array}{c}
\boldsymbol{X} \\
1
\end{array}\right]\right| \cdot\left|\left[\boldsymbol{p}^{3}\right]^{T}\left[\begin{array}{c}
\boldsymbol{X} \\
1
\end{array}\right]\right| .
\end{gathered}
$$

A finite difference approximation of the derivatives in the nonlinear system of equation (7) for the motion increment $\left(d u^{k}, d v^{k}\right)$ at $k$ iteration is given by

$$
\left(\psi_{D}^{\prime}\right)_{i}^{k}\left(\left(S_{11 i}^{k} d u_{i}^{k}+S_{12 i}^{k} d v_{i}^{k}+S_{13 i}^{k}\right)+\alpha\left(T_{11 i}^{k} d u_{i}^{k}+T_{12 i}^{k} d v_{i}^{k}+T_{13 i}^{k}\right)\right)-
$$




$$
\begin{aligned}
& -\beta \sum_{l=x, y} \sum_{j \in N_{l}(i)} \frac{\left(\psi_{s}^{\prime}\right)_{i}^{k}+\left(\psi_{s}^{\prime}\right)_{j}^{k}}{2} \frac{u_{j}^{k}+d u_{j}^{k}-u_{i}^{k}-d u_{i}^{k}}{\left(h_{l}^{k}\right)^{2}}+ \\
& +\gamma\left(\psi_{X}^{\prime}\right)_{i}^{k}\left(\left(a^{2}\right)_{i}^{k}\left(u_{i}^{k}+d u_{i}^{k}\right)+\left(q_{1}\right)_{i}^{k}\right)=0, \\
& \left(\psi_{D}^{\prime}\right)_{i}^{k}\left(\left(S_{12 i}^{k} d u_{i}^{k}+S_{22 i}^{k} d v_{i}^{k}+S_{23 i}^{k}\right)+\alpha\left(T_{12 i}^{k} d u_{i}^{k}+T_{22 i}^{k} d v_{i}^{k}+T_{23 i}^{k}\right)\right)- \\
& -\beta \sum_{l=x, y} \quad \sum_{j \in N_{l}(i)} \frac{\left(\psi_{S}^{\prime}\right)_{i}^{k}+\left(\psi_{S}^{\prime}\right)_{j}^{k}}{2} \frac{v_{j}^{k}+d v_{j}^{k}-v_{i}^{k}-d v_{i}^{k}}{\left(h_{l}^{k}\right)^{2}}+ \\
& +\gamma\left(\psi_{X}^{\prime}\right)_{i}^{k}\left(\left(a^{2}\right)_{i}^{k}\left(v_{i}^{k}+d v_{i}^{k}\right)+\left(q_{2}\right)_{i}^{k}\right)=0 .
\end{aligned}
$$

So matrix form of equation system (8) for each pixel is given by

$$
\left(\begin{array}{l}
d u^{k, n+1} \\
d v^{k, n+1}
\end{array}\right)=\left(\begin{array}{l}
M_{11}^{k, n} M_{12}^{k, n} \\
M_{12}^{k, n} M_{22}^{k, n}
\end{array}\right)^{-1}\left(\begin{array}{l}
r u^{k, n} \\
r v^{k, n}
\end{array}\right)
$$

with matrix entries

$$
\begin{aligned}
& M_{11}^{k, n}=\left(\psi_{D}^{\prime}\right)_{i}^{k, n}\left(S_{11 i}^{k, n}+\alpha T_{11 i}^{k, n}\right)+\beta \sum_{l=x, y} \sum_{j \in N_{l}(i)} \frac{\left(\psi_{S}^{\prime}\right)_{i}^{k, n}+\left(\psi_{S}^{\prime}\right)_{j}^{k, n}}{2\left(h_{l}^{k}\right)^{2}}+ \\
&+\gamma\left(\psi_{X}^{\prime}\right)_{i}^{k, n}\left(a^{2}\right)_{i}^{k}, M_{22}^{k, n}=\left(\psi_{D}^{\prime}\right)_{i}^{k, n}\left(S_{22 i}^{k, n}+\alpha T_{22 i}^{k, n}\right)+\beta \sum_{l=x, y} \sum_{j \in N_{l}(i)} \frac{\left(\psi_{S}^{\prime}\right)_{i}^{k, n}+\left(\psi_{S}^{\prime}\right)_{j}^{k, n}}{2\left(h_{l}^{k}\right)^{2}}+ \\
&+\gamma\left(\psi_{X}^{\prime}\right)_{i}^{k, n}\left(a^{2}\right)_{i}^{k}, \\
& \quad u^{k, n}=-\left(\psi_{D}^{\prime}\right)_{i}^{k, n}\left(S_{13 i}^{k, n}+\alpha T_{13 i}^{k, n}\right)+ \\
&+\beta \sum_{l=x, y} \sum_{j \in N_{l}^{-}(i)} \frac{\left(\psi_{S}^{\prime}\right)_{i}^{k, n}+\left(\psi_{S}^{\prime}\right)_{i}^{k, n}}{2} \frac{u_{j}^{k}+d u_{j}^{k, n+1}-u_{i}^{k}}{\left(h_{l}^{k}\right)^{2}}+ \\
&+\sum_{l=x, y} \sum_{j \in N_{l}^{+}(i)} \frac{\left(\psi_{S}^{\prime}\right)_{i}^{k, n}+\left(\psi_{S}^{\prime}\right)_{i}^{k, n}}{2} \frac{u_{j}^{k}+d u_{j}^{k, n}-u_{i}^{k}}{\left(h_{l}^{k}\right)^{2}}+ \\
&+\gamma\left(\psi_{X}^{\prime}\right)_{i}^{k, n}\left(\left(a^{2}\right)_{i}^{k} u_{i}^{k}+\left(q_{1}\right)_{i}^{k}\right), \\
& \quad r v^{k, n}=-\left(\psi_{D}^{\prime}\right)_{i}^{k, n}\left(S_{23 i}^{k}+\alpha T_{23 i}^{k, n}\right)+ \\
&+\sum_{l=x, y} \sum_{j \in N_{n}^{-}(i)} \frac{\left(\psi_{S}^{\prime}\right)_{i}^{k, n}+\left(\psi_{S}^{\prime}\right)_{i}^{k, n} \frac{v_{j}^{k}+d v_{j}^{k, n+1}-v_{i}^{k}}{2}+}{\left(h_{l}^{k}\right)^{2}} \\
&+\beta \sum_{l=x, y} \sum_{j \in N_{n}^{+}(i)} \frac{\left(\psi_{S}^{\prime}\right)_{i}^{k, n}+\left(\psi_{S}^{\prime}\right)_{i}^{k, n}}{2} \frac{v_{j}^{k}+d v_{j}^{k, n}-v_{i}^{k}}{\left(h_{l}^{k}\right)^{2}}+ \\
&+\gamma\left(\psi_{X}^{\prime}\right)_{i}^{k, n}\left(\left(a^{2}\right)_{i}^{k} v_{i}^{k}+\left(q_{2}\right)_{i}^{k}\right) .
\end{aligned}
$$

Here $(i, j)$ - a pixel coordinate, $\left(d u^{k, n+1}, d v^{k, n+1}\right)$ - the motion vector between two images, $k$-index of fixed point iterations method, $\psi_{D}^{\prime}-$ a robustness factor in optical flow 
data term, $\psi_{S}^{\prime}$ - optical flow smoothness term diffusivity, $\psi_{X}^{\prime}$ - stereo constraint penalty function, $N_{n}^{+}:=\left\{j \in N_{n}(i) \mid j>i\right\}-$ means the set of pixels that have yet to be processed in current SOR iteration, $N_{n}^{-}:=\left\{j \in N_{n}(i) \mid j<i\right\}$ - denotes the set of already computed pixels, $n-$ is the iteration index of the point coupled SOR solver, $h$ - rectangular pixel grid size.

\subsection{Stereo constraint}

The scene coordinates obtained by functional (4) of $\mathbf{X}$ minimizing and leads to least squares method

$$
\left[\begin{array}{c}
{\left[x \boldsymbol{p}_{0}^{3}-\boldsymbol{p}_{0}^{1}\right]^{T}} \\
{\left[y \boldsymbol{p}_{0}^{3}-\boldsymbol{p}_{0}^{2}\right]^{T}} \\
{\left[(x+u) \boldsymbol{p}^{3}-\boldsymbol{p}^{1}\right]^{T}} \\
{\left[(y+v) \boldsymbol{p}^{3}-\boldsymbol{p}^{2}\right]^{T}}
\end{array}\right]\left[\begin{array}{c}
\boldsymbol{X} \\
1
\end{array}\right]=0
$$

The system of equations (7) and (10) is solved in the iterative framework by iterating between the optical flow $\mathbf{w}=(u, v)$ computation and scene structure $\mathbf{X}$ solving.

\subsection{Algorithm of stereo vision-based optical flow computation}

We also traditionally apply the coarse-to-fine technique by image pyramid building for the variational approach of optical flow computation.

Input data: $I_{1} ; I_{2}$

Create images pyramid;

while $s<$ max_level do //pyramid loop

while iter $<k$ _iters do //external linearization loop

// image warping iterations

// current pyramid level images $I_{1}^{s}$ and $I_{2}^{s}$ derivatives

$I_{x}, I_{x x}, I_{y}, I_{x y}, I_{z}, I_{z x}, I_{z y}$;

while iter < l_iters do//internal linearization loop

foreach col, row in Images

$[0$, nRows -1$] \times[0, n$ Cols -1$]$ do

// optical flow vector derivatives

$u_{x}, u_{y}, v_{x}, v_{y}$

// optical flow penalty functions

$\psi_{D}^{\prime}, \psi_{s}^{\prime}$;

//optical flow tensors

$S, T$;

// stereo pair structure coefficients

$a, b, q$;

// stereo pair structure penalty function

$\psi_{X}^{\prime}$;

// joint tensors 
$M_{11}, M_{12}, M_{21}, M_{22}, r u, r v$

// Successive Over-Relaxation

while iter $<m_{-} S O R \_$iters do //SOR loop

$$
\left(d u^{n+1}, d v^{n+1}\right):=\operatorname{SOR}\left(d u^{n}, d v^{n}\right) ;
$$

end while // end SOR $m$

// image $I_{2}$ warping by $(d u, d v)$

$I_{2}^{s}:=\operatorname{warp}\left(I_{2}^{s},(d u, d v)\right) ;$

end while // end $l$

// optical flow vector update

$u:=u+d u, v:=v+d v$;

// projection matrix update

$P=K[R \mid \mathbf{t}]$

// stereo pair structure minimization

solve least square $\mathbf{X}=(X, Y, Z)$;

end while //end $k$

// optical flow on next pyramid level interpolation

$\left(u^{s+1}, v^{s+1}\right):=\operatorname{bilinear}\left(\left(u^{s}, v^{s}\right), \eta\right)$

/lend $s$

\section{Results}

We implemented our method on Xilinx Zynq SoC heterogeneous platform [6]. High-efficient variational optical flow computation was achieved by its hardware FPGAs-accelerator implementation and subtask of least squares method of scene coordinates optimization was done on ARM cortex-A9 software module [7].

Table 1. BAD2.0 \% Middlebury Stereo Dataset Out-Noc metric.

\begin{tabular}{|c|c|c|c|c|}
\hline \multicolumn{2}{|c|}{ Scene } & Proposed & NOSS & SGBM1 \\
\hline $\mathrm{N}$ & Name & \multicolumn{3}{|c|}{ BAD 2.0\% } \\
\hline 1 & Adirondack & 5.81 & 3.51 & 29.3 \\
\hline 2 & Shelves & 15.1 & 27.5 & 49.9 \\
\hline 3 & Playroom & 23.15 & 17.5 & 39.7 \\
\hline 4 & Playtable & 37.5 & 8.73 & 45.6 \\
\hline 5 & Vintage & 28.69 & 8.97 & 49.0 \\
\hline
\end{tabular}

To evaluate the optical flow we used public available Middlebury Stereo Datasets [8] with ground truth and compared our approach with existing state-of-art methods. For an image input size of quarter resolution up to $750 \times 500$ pixels our approach computes optical flow frame by FPGA-accelerated successive over-relaxation method at $\approx 50 \mathrm{~ms}$. Adding the ARM-processing time of the least squares $3 \mathrm{D}$ points estimation at $\approx 30 \mathrm{~ms}$, the entire computation is done at approximately $80 \mathrm{~ms}$.

We show optical flow (disparity) evaluation results in Figure 1 for sample pairs with the error metric measuring BAD2.0 \% - the percentage of erroneous pixels in non-occluded areas whose disparity error is greater than 2.0 pixels. 
We also present a comparison of the three methods in Table 1. From the results, our approach has slightly low accuracy than NOSS method, which is a state-of-art network learning method and better accuracy than the popular OpenCV SGBM1 method.

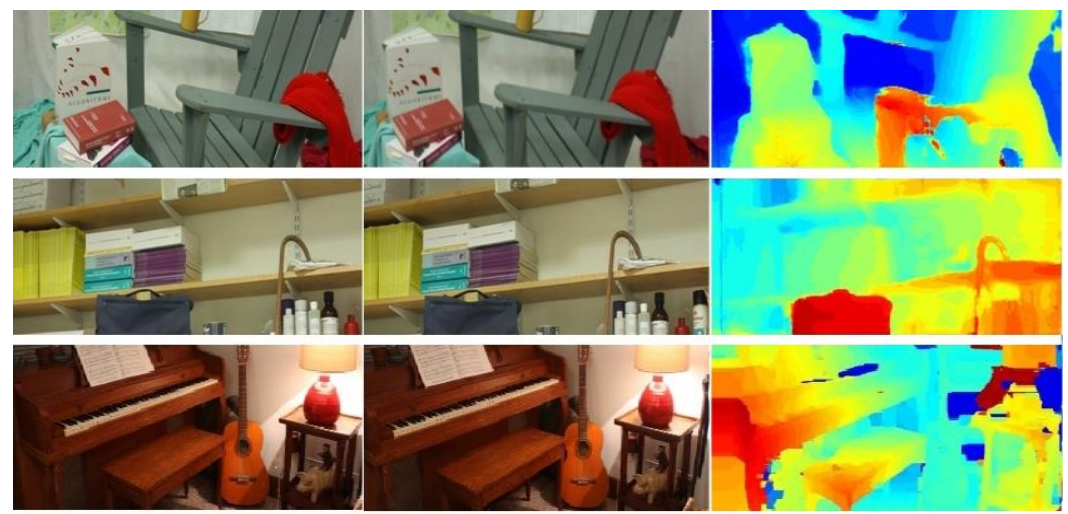

Fig. 1. Optical flow estimation results on the Middlebury Stereo Datasets (Adirondack, Shelves and Piano images) for proposed method.

\section{Conclusion}

We have shown that the variational framework of joint energy functional minimization consisting of an optical flow constraint and camera pose constraint helps to improve the computation of optical flow. Benefits are due to including camera pose constraint inside of basic optical flow PDE numerical approximation technique. We also achieved comparable results with existing variational and network learning-based methods for some popular benchmarks.

\section{References}

1. Brox T, Bruhn A, Papenberg N and Weickert J 2004 LNCS ECCV (Berlin: Springer) 25

2. Roxas M, Oishi T 2018 IEEE Winter Conference on Applications of Computer Vision

3. Hartley R and Zisserman A 2000 Multiple View Geometry in Computer Vision (Cambridge, UK: Cambridge University Press)

4. Valgaerts L, Bruhn A, Mainberger M, Weickert J 2012 International Journal of Computer Vision 96(2) 212

5. Bruhn A and Weicker J 2005 Proc. 10th IEEE Int.Conf., Computer Vision 749

6. Ustukov D, Muratov Y, Nikiforov M, Gurov V 2016 Mediterranean Conference on Embedded Computing (MECO 2016)

7. Belyakov P, Nikiforov M 2018 Proc. in IEEE East - West Design and Test Symposium (EWDTS 2018) p 129

8. Middlebury Stereo Dataset http://vision.middlebury.edu/stereo/eval3/MiddEval3newFeatures.html 